\title{
Conocimiento en colabor: Reflexiones y posibilidades para la construcción de paz
}

\author{
Knowledge in collaboration: Reflections \\ and possibilities for the construction of peace
}

\author{
Adriana Arroyo Ortega ${ }^{1}$ \\ adriana.arroyo.ortega1@gmail.com \\ Sara Victoria Alvarado Salgado 2 \\ alvarado.s@gmail.com
}

\begin{abstract}
Resumen
Este artículo desarrolla un análisis acerca de las cuestiones metodológicas y epistemológicas que se encuentran en la investigación en ciencias sociales, a partir de diversas reflexiones en torno al abordaje de las perspectivas de la decolonialidad y la colabor, contemplándole desde sus fortalezas y posibilidades para una investigación que contribuya al agenciamiento de niños y jóvenes. En un primer momento se explicitan algunas condiciones de la investigación en América Latina, posteriormente se centra en generar una deliberación sobre el conocimiento, la construcción del mismo, desde el enfoque decolonial, para finalizar con la pregunta por la investigación en colabor como aporte concreto para minimizar las asimetrías investigativas de cara a la construcción de paz, clausurando con el cierre de lo expresado.
\end{abstract}

\section{Palabras clave}

Investigación, conocimiento, niñez, juventud, paz. (Tesauro de la UNESCO)

Forma sugerida de citar:
Arroyo Ortega, Adriana, \& Alvarado Salgado, Sara Victoria (2016). Conocimiento en colabor: Reflexiones y posibilidades para la construcción de paz. Universitas, XIV (25), pp. 121-148.

1 Administradora en Salud, Magíster en Educación y Desarrollo Humano, Doctora en Ciencias Sociales, Niñez y Juventud de la alianza Universidad de Manizales - CINDE. Profesora-Investigadora de la Universidad de Manizales y del Centro Internacional de Educación y Desarrollo Humano-Cinde.

2 Psicóloga, Doctora en Educación Nova University - CINDE. Directora del Centro de Estudios Avanzados en Niñez y Juventud CINDE - Universidad de Manizales, Colombia. Coordinadora del programa grupos de trabajo CLACSO. 


\begin{abstract}
This article develops an analysis on methodological and epistemological issues found in research in social sciences from various reflections on the approach prospects decoloniality and collaborated, watching him from their strengths and possibilities for research contributing to the assemblage of children and youth. At first some research conditions in Latin America are explained later is focused on generating a discussion about knowledge, its construction, from the decolonial approach, ending with the question of research collaborated as a concrete contribution to minimize asymmetries facing investigative peacebuilding, ending with the closing of the statement.
\end{abstract}

Keywords

Research, knowledge, childhood, youth, peace.

\title{
Introducción
}

Vivimos un tiempo lleno de complejidades, de profundas inequidades y desastres ecológicos y humanos, que tiene como telón de fondo un escenario de capitalismo salvaje y de incumplimiento de las promesas de la modernidad, pero en las que paradójicamente se sustenta aun la idea del progreso y la relación saber-poder, así como las atmósferas de violencias y vulneraciones que muchos seres -humanos y no humanos- vienen sufriendo. Teniendo en cuenta este marco, es necesario preguntarse por el conocimiento, sus implicaciones, la forma en que este se produce y cómo en esa producción se ha incluido o no a los niños, niñas y jóvenes, así como los aportes posibles de la misma a la construcción de paz.

Reflexionar sobre la forma en que se ha venido construyendo el conocimiento es crucial, especialmente cuando en muchos casos el llamado ha sido a la distancia, al objetivismo metodológico, que sitúa al investigador de manera aséptica frente a realidades tan complejas como las existentes en América Latina, cargadas de injusticias, violencias y silenciamientos históricos y en que se termina objetivando a los niños, niñas y jóvenes, desconociendo sus posibilidades como seres humanos concretos, que aportan con sus voces de manera crítica y transformadora a la realidad social de la que son parte. 
De todas maneras y aunque se reconoce que las prácticas científicas no son homogéneas y en las mismas coexisten agendas, énfasis y estrategias diversas, pensar en abordajes epistemológicos como los que se plantearan a continuación, ofrecen importantes aportes para vislumbrar acercamientos más situados a los niños, niñas y jóvenes, sus transformaciones y realidades, así como a la vez se propicia la interpelación de las propias prácticas y conocimientos construidos desde perspectivas eurocéntricas y patriarcales, en aras de que desde la investigación misma, puedan generarse pistas para la descolonización y la construcción de múltiples paces que incorporen las voces y experiencias de los jóvenes, niñas y niños.

Es fundamental entonces que los investigadores/as puedan comenzar a construir marcos analíticos diversos que les involucren también a ellos, en relación directa con el conocimiento y los actores sociales con los que los construyen, en aras de propiciar nuevas y más potentes visibilidades y audibilidades de la especificidad de las experiencias y conocimientos de niños, niñas y jóvenes en América Latina, reconociendo que en las mismas se pueden encontrar aportes y anclajes valiosos para la construcción de paz; que en suma, ellos y ellas tienen un pensamiento sobre sí mismos y el mundo que nos pueden llevar no sólo a comprender sus posicionamientos como sujetos, sino también a generar nuevas formas de acercamiento y transformación a las problemáticas sociales existentes.

En este texto se busca precisamente crear algunas pistas reflexivas sobre estos temas a partir de la propia experiencia investigativa de las autoras y el trabajo con niñas, niños y jóvenes y de manera singular, la conversación sostenida con una joven afrodescendiente perteneciente a un colectivo de la ciudad de Medellín.

\section{La pregunta epistemológica desde América Latina: EI Sur con un contexto específico}

Proponer la reflexión sobre las ciencias sociales desde los contextos de sufrimiento, violencia y miedo que atraviesan muchos de los territorios latinoamericanos, implica en primer lugar reconocer que las ciencias mismas han venido transitando por circunstancias complejas, ya que como lo expresa Santos (2009): 
En el dominio de la organización del trabajo científico, la industrialización de la ciencia produjo dos efectos principales. Por un lado, la comunidad científica se estratificó, las relaciones de poder entre los científicos se tornaron más autoritarias y desiguales y la abrumadora mayoría de los científicos fue sometida a un proceso de proletarización en el interior de los laboratorios y de los centros de investigación. Por otro lado la investigación capital - intensiva (basada en instrumentos caros y raros) torno imposible el acceso al libre equipamiento, lo que contribuyó a ensanchar la brecha en términos de desarrollo científico y tecnológico entre países centrales y países periféricos (p. 39).

Situaciones que van dejando por fuera de la producción de la ciencia las voces y perspectivas de países llamados emergentes, pero además va privilegiando una única forma de construir conocimiento en la que las narrativas de los actores sociales, sobre todo niños, niñas y jóvenes, se diluyen en los regímenes del discurso especializado.

No significa esto que se desdeñe el conocimiento científico, sino que se hace necesario interpelarlo, generar preguntas y reconocer la importancia del diálogo y la escucha en los procesos de investigación, pero también la reflexión sobre las certezas instaladas en las ciencias y en los/las científicos/as, lo cual tiene un sentido especialmente importante cuando se es consciente de cómo a pesar de las promesas de paz perpetua de la modernidad (Kant, 1975; 2005), se han materializado cada vez más guerras y conflictos de todo tipo en el planeta, y como a pesar del supuesto progreso y bienestar social para todos y todas, millones de personas, esencialmente niñas, niños y jóvenes, siguen sumidas en la pobreza y con grandes inequidades sociales.

Al respecto basta con revisar el informe del PNUD sobre Desarrollo $\mathrm{Hu}-$ mano 2013:

Se calcula que alrededor de 1,57 mil millones de personas, o más del 30\% de la población de los 104 países analizados, viven en condiciones de pobreza multidimensional, un índice que incluye tanto la cantidad como la intensidad de carencias simultáneas sufridas por las personas en salud, educación y nivel de vida. En muchos de los países del Sur con crecimiento acelerado, la población que vive en condiciones de pobreza multidimensional supera a la que sufre pobreza de ingresos. A su vez, el grado de desigualdad en los ingresos aumenta en muchos países (p. 13).

Esto da cuenta de dificultades históricas y estructurales en estos aspectos que no se pueden desconocer, evidenciando las múltiples caras de la 
opresión, de la pobreza y la discriminación, especialmente con el señalamiento sobre como la ciencia ha partido de la formulación de preguntas sobre la vida desde la perspectiva de hombres blancos, occidentales y de cierta clase social (Harding, 1998), lo que deja por fuera del análisis y preguntas de la ciencia a los niños, niñas y jóvenes, así como a los indígenas, afros, gay, transgéneros, y en general a los grupos sociales que sistemáticamente han sido excluidos, especialmente a los que Santos (2009) y otros denominan periféricos o del Sur.

Reconocer la importancia de las experiencias infantiles, juveniles, racializadas, generizadas, subalternizadas y del Sur, se configura como un recurso indispensable para tener un panorama más completo del mundo y generar un análisis social más pertinente, que nos lleve como lo plantea Santos (2006, p. 105) a "crear las condiciones para una justicia global a partir de la imaginación democrática", lo que se configura como substancial en las condiciones actuales de los contextos latinoamericanos. Esta actitud crítica no puede dejar tampoco de lado la posibilidad de generar reflexión sobre las mismas ciencias sociales y como su conformación y los marcos epistemológicos que las definen, están asentadas igualmente sobre visiones eurocéntricas del mundo, que trazan rutas de un conocimiento traído desde otras latitudes, que desconoce en muchos casos las propias realidades de cada uno de los países, que no son necesariamente homogenizables.

Aprender con el Sur y desde el Sur como metáfora, con los niños, niñas y jóvenes, involucra en alguna medida:

Conocerse a sí mismas y al mundo a través del conocimiento-emancipación, recurriendo a una retórica dialógica y a una lógica emancipadora; por otro lado capaz de concebir y desear alternativas sociales basadas en la transformación de relaciones de poder en relaciones de autoridad compartida y en la transformación de los órdenes jurídicos despóticos en ordenes jurídicos democráticos (Santos, 2003, p. 393).

Pero a la vez implica realizar una crítica a la ciencia, a la forma de producción de conocimiento que hegemónicamente se ha construido, reconociendo que "la ciencia moderna, además de moderna, es también occidental, capitalista y sexista. Esta triple adjetivación no es circunstancial" (Santos, 2003, p. 95) porque históricamente la ciencia ha combinado el racismo con el sexismo, lo que constituye dobles o triples exclusiones para los sujetos a 
los que se les atribuye las categorías raciales o de género, que estarían por fuera de los privilegios socialmente establecidos.

Lo que hace necesaria la producción de conocimiento no neutral, "conocimiento basado en una perspectiva ética sobre la inequidad y desigualdad entre hombres y mujeres" (Carosio, 2010, p. 5) que permita que la academia y la sociedad reflexionen sobre cómo lo producen, para qué y en qué contextos y cómo este transforma o no las relaciones sociales inequitativas. En esa visión es importante poner en cuestión el asunto de los procesos de racialización que han perpetuado hasta hoy las condiciones de exclusión y subordinación de las niñas, niños y jóvenes afrodescendientes e indígenas, a quienes se le ha rechazado de manera sistemática como parte de la sociedad en algunos países, se les ha situado en el lugar de lo sexual o la ignorancia, sin reconocer en ellas y ellos su valía como sujetos y como seres humanos pensantes y sintientes, que tienen mucho que aportar a los procesos sociales y en la construcción de conocimiento.

El racismo soterrado y excluyente, asentado en prejuicios histórica y culturalmente construidos, debe ser develado y puesto en evidencia, explicitando además que las niñas y jóvenes racializadas pueden sufrir dobles y tiples discriminaciones: por ejemplo, ser joven, afro y pobre en un continente tan inequitativo como el latinoamericano.

El documento de la CELADE realizado por Del Popolo F., López M. y Acuña M. (2008, p. 108), lo expresa claramente al decir:

En la mayoría de los países, los jóvenes indígenas y afrodescendientes enfrentan grandes dificultades en el acceso y permanencia en el sistema educativo, y se encuentran entre los grupos más desfavorecidos. La situación de las niñas y jóvenes es particularmente preocupante, pues pocas culminan la educación primaria o logran pasar a la secundaria, siendo en muchos casos analfabetas funcionales y monolingües.

Ese racismo histórico y la exclusión que lo acompaña no ha desaparecido y minimiza los aportes de las afrodescendientes, indígenas, raizales y en general de las poblaciones que son diferentes a los estándares de lo blanco, lo que ha perpetuado y exacerbado la violencia machista y racista. En el caso de los afrodescendientes en Colombia:

En 2004, por ejemplo, el Relator Doudou Diène señalaba en su informe de visita a Colombia que el gobierno reconocía que el $82 \%$ todavía vive sin que 
se subvengaran sus necesidades básicas (abastecimiento de agua, luz eléctrica, saneamiento, entre otras); la tasa de analfabetismo de esta población era tres veces superior a la del resto ( $43 \%$ en el campo y $20 \%$ en la ciudad, en comparación con el $23,4 \%$ y el 7,3\% para el resto), y que de cada 100 jóvenes afrocolombianos apenas dos tenían acceso a estudios superiores. Asimismo, el Relator consignaba en su informe que la tasa de mortalidad infantil entre los afrocolombianos era del $151 \%$, mientras que el promedio nacional era del 39\%; el 76\% vivía en condiciones de extrema pobreza y el $42 \%$ estaba sin empleo (Antón J., Bello Á., Del Popolo F., Paixão M. \& Rangel M., 2009).

Situaciones que no ha tenido cambios sustanciales a lo largo de estos años y que dan cuenta de las dificultades de acceso en general para la población afrodescendiente y en particular para las jóvenes, especialmente en la educación superior y en el trabajo; barreras que no siempre pueden verse reflejadas en indicadores estadísticos pero que limitan de manera clara las posibilidades de ingreso, producción de conocimiento y calidad de vida para ellas.

La deliberación sobre el género, el colonialismo y el cruce con la discriminación étnica y el invento de las razas, como forma de perpetuar la opresión y la pobreza de algunos pueblos, ha situado contextualmente algunos aspectos del pensamiento latinoamericano, pero es necesario que se amplíe cada vez más el cuestionamiento a todas las formas de negación de la diferencia y homogenización de los sujetos, pero también cuál ha sido el papel de las ciencias sociales, de los científicos en torno a estas realidades. Este cruce de la reflexión con los procesos de racialización, se amplía con la crítica a la matriz heteronormativa que disminuye las posibilidades de elección y construcción subjetiva, explicitando que en muchas de las sociedades latinoamericanas contemporáneas todavía hay grandes temores hacia las jóvenes, los jóvenes y niños que van más allá de la heterosexualidad establecida y cómo aunque han ido generándose avances en torno a investigaciones al respecto, aun se tiene desde la producción de conocimiento no sólo una deuda en torno a estos temas con ellos y ellas, sino que además es necesario discurrir sobre la postura ética y política que los investigadores/as tienen y asumen en la producción de conocimiento sobre estas temáticas.

Es importante entonces el que se puedan contar las historias no contadas, las que han sido invisibilizadas e incluso estigmatizadas, y en ese entramado la descolonización no sólo es una propuesta epistemológica, sino también política, en la que es esencial resaltar que precisamente esta visión ha tenido en América Latina un gran epicentro desde las perspectivas de pensa- 
doras/es como Walsh, Schiwy y Castro Gómez (2002) Rivera Cusincanqui (2010) o Mignolo (2003) entre muchas otras y otros; articulando una pensamiento desde la racialización, la clase y la sexualidad como asuntos centrales en una visión geopolítica, deconstruyendo y resignificando estas y otras categorías socialmente construidas.

Cuestionar la relación saber-poder es un eje central del proceso decolonial en el que autores como Quijano (2014) ponen en evidencia que categorías como la raza que han sido naturalizadas, son socialmente construidas y con intereses específicos para generar una noción de inferioridad en la recién descubierta América. Esto no sólo tiene anclajes teóricos, sino que como lo establece una joven de un colectivo de Medellín, se percibe para muchos de ellos y ellas en la cotidianidad de la vida y en las prácticas sociales:

A veces nos quisieran hacer creer mentiras, que lo único que cuenta es tu esfuerzo, pueda que para algunas personas sea así ¿No? Digo, pueda que para algunas personas su esfuerzo valga muchísimos y a través de él puedan hacer todo lo que quieran, pero a la larga no es sólo el esfuerzo, porque para llegar a ser eso que quieres ser sólo desde ti, tienes que renunciar a un montón de otras cosas (...) y es como la cuestión de que, para, a pesar de tu esfuerzo, para, a veces para tener un lugar que llaman privilegiado, tienes que renunciar a cosas, que tiene que ver como contigo, con tu identidad ¿Cierto? (Comunicación personal Yacila 2015).

Ese renunciar a lo que se constituye como elemento central de la producción identitaria de los sujetos afrodescendientes que expone Yacila, en aras de la adaptación a los estándares culturales definidos socialmente y en los que en muchas ocasiones no logran interlocutar y ser vistos en la expansión de sus potencialidades, habla precisamente de la necesidad de iniciar procesos de descolonización que puedan articular las prácticas sociales y los procesos investigativos del actual campo de producción de conocimientos.

Esta descolonización implica el rescate de las diversas propuestas epistemológicas y políticas que desde los mismos jóvenes, niñas y niños indígenas y afrodescendientes se han generado, resistiendo la universalización y homogenización y valorando el pensamiento propio. La descolonización no sólo es un asunto cognitivo, es necesario que atraviese los cuerpos, que como opción política implique reflexionar sobre el propio conocimiento, sobre "nuestros imaginarios, nuestros cuerpos, nuestras sexualidades, nuestras formas de actuar y de ser en el mundo y que crea una especie de "cimarro- 
naje" intelectual, de prácticas sociales y de la construcción de pensamiento propio de acuerdo a experiencias concretas" (Curiel, 2009 p. 3).

Desde el cuestionamiento constante como lo esboza Curiel (2009, p. 3) al "sujeto único, al eurocentrismo, al occidentalismo, a la colonialidad del poder, al tiempo que reconoce propuestas como la hibridación, la polisemia, el pensamiento otro, subalterno y fronterizo" se permite a la vez que emerja la necesidad de pensarse y repensarse, generando la reflexión sobre la manera en que se interconectan el sexismo, el racismo y el capitalismo, como una única matriz de dominación que puede afectar a todos los sujetos centrándose en las/los jóvenes, niños y niñas racializados. Esta crítica necesaria a lo socialmente aceptado, naturalizado y establecido, a la universalidad y homogenización, a lo etnocentrico y heterocentrico, es fundamental en aras de visibilizar la voz, narrativas y experiencias de las jóvenes, los jóvenes, niños y niñas, en su potencia desde las realidades sociales, pero también desde la producción de conocimiento científico.

Por esto como lo explica Curiel (2009, p. 7) debemos zafarnos de esa "dependencia intelectual euronorcéntrica, lo cual no niega que sean referentes teóricos importantes" pero a los que es necesario interpelar, así como explicitar las producciones propias que pueden ser puestas en dialogo con las de otros contextos, reconociendo además como lo esboza Rivera Cusicanqui (2010, p. 19) "la idea de que en el presente de nuestros países continúa en vigencia una situación de colonialismo interno" que explica pero no justifica muchas de las realidades sociales, económicas y políticas de América Latina.

Esto coincide en alguna medida con lo expresado por Yacila frente a las consideraciones sobre las exclusiones y manifestaciones cotidianas de racismo que ella ha sufrido:

Hemos comprendido que mucha, mucho de la forma de pensar de la gente o ese racismo estructural que llaman, responde a... como a la forma en que nos educaron, y a nosotros nos educaron con esas divisiones, y así como a nosotros nos educaron haciéndonos pensar que éramos como la... como que están como en el nivel más inferior, por decirlo así, de la humanidad, a ellos los educaron también haciéndoles creer que nosotros estamos en el nivel, entonces, si bien mucha gente es consciente de sus actos racistas y los hace con toda la alevosía del caso, hay otra gente que no, hay otra gente que simplemente, que responde como a una lógica que tiene incorporada, ahí como una especie de chip, porque eso desde chiquitos, en la televisión y en todas les partes les dijeron que los negros eran como bruticos, como más feítos, como perezositos (Conversación personal Yacila 2015). 
Al respecto Carneiro (2009), expresa que las que podrían ser consideradas historias o reminiscencias del periodo colonial permanecen vivas en el imaginario social y adquieren nuevos ropajes y funciones, en un orden social supuestamente democrático que mantiene intactas las relaciones de género y las distribuciones raciales. Relaciones que no siempre son interrogadas ni cuestionadas y que generan divisiones sociales profundas a partir de la exclusión de estos grupos y personas. En algunos países latinoamericanos como es el caso de México o Colombia se ha creído que se transforman las realidades sociales de discriminación y violencia contra las mujeres, los niños y niñas a partir de prohibiciones y leyes, pero no se han enfocado en las mutaciones culturales necesarias para generar una deconstrucción del machismo y el racismo como forma colonial de admiración al hombre blanco, y propiciar la generación de nuevas formas de ser hombres, que no implique la violencia hacia las mujeres o la consideración de ellas o de los niños, niñas y jóvenes racializados, como seres inferiores.

Enmarcadas dentro de lo que Alexander y Mohanty (2004), han definido como el desafío del "feminismo del tercer mundo", es necesario ubicarse en la necesidad de construir propuestas epistemológicas que descolonicen el conocimiento (Mohanty, 2008), que no sólo da cuenta de una realidad, sino que la construye permanentemente.

Esto además explicita una relación con la violencia moral, una violencia simbólica, cotidiana y rutinaria, que se inscribe en los relacionamientos y que genera mella en las subjetividades que las sufren, de manera particular las niñas y jóvenes, como lo explicita Segato (2003, p. 121).

El paralelismo entre el racismo automático y el sexismo automático, ambos sustentados por la rutinización de procedimientos de crueldad moral, que trabajan sin descanso la vulnerabilidad de los sujetos subalternos, impidiendo que se afirmen con seguridad frente al mundo y corroyendo cotidianamente los cimientos de su autoestima, nos devuelve al tema del patriarcado simbólico que acecha por detrás de toda estructura jerárquica, articulando todas las relaciones de poder y subordinación.

Este patriarcado simbólico y las violencias morales inscritas en el, se relacionan también con la producción de conocimiento y la manera en que aparecen o no reflexionados o visibilizados en los cánones científicos los procesos de exclusión, subordinación e intimidaciones de todo orden, que sufren cotidianamente los sujetos que han sido históricamente excluidos de 
los circuitos de la ciencia y la investigación y de manera particular las niñas, niños y jóvenes racializados.

\section{La reflexión decolonial en torno al conocimiento}

La reflexión epistemológica en torno a la producción de conocimiento ha sido una preocupación que se ha tenido desde distintas perspectivas teóricas y autores como (Kuhn, [1971] (2011); Popper, [1962] (2008); Lakatos, 1983, 1993; Habermas, 2002), entre muchos otros que han hecho importantes aportes al debate al respecto. Pero de maneras relativamente más recientes la pregunta que se ha hecho desde África o América Latina pasa por la manera en que puede enriquecerse dicha producción desde una perspectiva crítica que involucre a los conocimientos subalternizados y a los sujetos invisibilizados, en el reconocimiento del epistemicidio del que habla Santos (2009).

Como lo precisan Walsh, Schiwy y Castro Gómez (2002, p. 8) "la cuestión del poder del conocimiento y sus vínculos con los intereses del capitalismo y la historia colonial -el problema de las geopolíticas del conocimiento- es un tema que se empezaba a discutir con gran interés en América Latina a finales de los noventa" y sobre lo que se han generado encuentros, debates y distintas producciones académicas, especialmente desde la perspectiva que nos interesa explicitar en este artículo como lo es la decolonial.

Las investigadoras/es y pensadores/as que asumen esta postura teórica y política explicitan que no vivimos en un mundo descolonizado, sino que, por lo contrario, es necesario:

Trascender la suposición de ciertos discursos académicos y políticos, según la cual, con el fi n de las administraciones coloniales y la formación de los Estados-nación en la periferia, vivimos ahora en un mundo descolonizado y poscolonial. Nosotros partimos, en cambio, del supuesto de que la división internacional del trabajo entre centros y periferias, así como la jerarquización étnico-racial de las poblaciones, formada durante varios siglos de expansión colonial europea, no se transformó significativamente con el fin del colonialismo y la formación de los Estados-nación en la periferia. Asistimos, más bien, a una transición del colonialismo moderno a la colonialidad global, proceso que ciertamente ha transformado las formas de dominación desplegadas por la modernidad, pero no la estructura de las relaciones centroperiferia a escala mundial (Castro Gómez y Grofoguel, 2007, p. 13). 
Y en este marco de la colonialidad/modernidad diversos autores y movimientos sociales en América Latina han encontrado sentidos fundamentales para construir pensamiento y acción política resaltando lo étnico, el género y todas las potencialidades que desde el conocimiento situado se puede generar en este contexto particular. Pero también hay otras voces al respecto, como la de Rivera Cusicanqui (2010) quien establece una tensión al interior de la decolonialidad, confirmando que no es un movimiento teórico homogéneo, al realizar críticas a las estructuras universitarias que subyacen en dicha reflexión y el que en muchos casos no ve una ruta clara de construcción práctica más allá del discurso, y así dice:

Por razones obvias, y a medida que se agudiza la crisis de las universidades públicas en América Latina, el tipo de estructura que hemos descrito se presta muy bien al ejercicio del clientelismo como modo de dominación colonial. A través del juego del quién cita a quién, se estructuran jerarquías y acabamos teniendo que comer, regurgitado, el pensamiento descolonizador que las poblaciones e intelectuales indígenas de Bolivia, Perú y Ecuador habíamos producido independientemente (p. 66).

Una crítica desde luego valida que no sólo atañe a la teoría decolonial y a sus principales exponentes, sino que se extiende de forma certera a la producción de conocimiento, a la circulación del mismo y a cómo algunos autores/as son considerados parte del canon de los que deben ser leídos y otros no, a la definición de los procesos y ritmos académicos en los programas de formación posgraduada y a las presiones economicistas de los sistemas de ciencia y tecnología, pero sobre todo a la visión que se tiene en torno a lo educativo, lo pedagógico y cómo entran ahí los jóvenes, niños y niñas.

Reconociendo el valor que puede tener la crítica de Rivera Cusicanqui (2010) y de algunos colectivos sociales a la teoría decolonial, es importante explicitar la fuerza que al debate desde América Latina le ha aportado esta visión, y no sólo a los movimientos sociales, también a los mismos intelectuales latinoamericanos ya que como lo explicita Fanon (1963):

El intelectual colonizado asiste, en una especie de auto de fe, a la destrucción de todos sus ídolos: el egoísmo, la recriminación orgullosa, la imbecilidad infantil del que siempre quiere decir la última palabra. Ese intelectual colonizado, atonizado por la cultura colonialista, descubrirá igualmente la consistencia de las asambleas de las aldeas, la densidad de las comisiones del pueblo, la extraordinaria fecundidad de las reuniones de barrio y de célula (p. 35). 
Esa reflexión, esos descubrimientos comportan entonces nuevas posibilidades, nuevos retos que aparten a los académicos de la perspectiva colonizadora que los ha habitado históricamente, llevándolos a la reflexión ética y epistemológica sobre sus procesos investigativos y de cómo el conocimiento es una construcción colectiva que se genera con los sujetos, niños, niñas y jóvenes, pero sobre todo el que:

Los académicos tenemos que reconocer la existencia de hegemonía (tales como la institución académica) y a la vez trabajar para su disolución o democratización. Denunciar el poder de la academia no es suficiente. La academia necesita reconocer que los nuevos medios están abriendo otros espacios del pensar y el debatir (Schiwy, 2002, p. 128).

Esos nuevos espacios implican reconocer también la producción de conocimiento desde los jóvenes, niñas y niños con rostro y cuerpo que tienen apuestas profundas por la transformación del mundo:

Las causas políticas son principalmente dos ¿Cierto? En un principio fue una, la raza yo sólo veía todo desde la raza, la raza, la raza, la raza, porque en Afro** de cierta manera aprendí que muchas de las limitaciones que yo había tenido en la vida eran porque era negra, siempre, y ya. Luego empezó a aparecer el asunto del género ¿No? Y eso lo descubrí en Afro**, no de la misma manera que descubrí el asunto de la raza, sino como... es que no sé cómo decirlo, como desde otra dimensión, más como de... desde la misma experiencia, estamos en un espacio político donde hablabas de una reivindicación política que es en cierta manera, que es no, que es libertaria, pero también siento que hay como cierto dominio, cierta jerarquía (Yacila, comunicación personal 2015).

Estas circunstancias ontológicas-existenciales de generización y racialización, vienen siendo interrogadas en aras de su transformación o interrupción, por parte de los mismas jóvenes, niños y niñas en sus distintos movimientos y apuestas. Casos concretos como el Consejo Infantil y Adolescente del municipio de Medellín o el trabajo de jóvenes como las del colectivo Somos Cimarrón o del grupo de mujeres jóvenes del Cepafro, dan cuenta de las búsquedas de muchas, muchos de ellas y ellos por interpelar o incluso superar las categorías socialmente construidas y colonialmente impuestas, asociadas a la peyorativización de la diferencia y a tecnologías otroficantes centradas en la inferiorización. 
Como lo esboza Mayer (2015, p. 31) "en amplios sectores de la población persisten patrones de relaciones de género mucho más desiguales que son llevados a los diversos ámbitos donde esos actores interactúan: la familia, la escuela, el trabajo y los espacios de socialización, entre otros (Andrade, 2002)", por lo que es imperativo problematizar las formas en que se están estructurando las relaciones de poder y diferenciación en dichos espacios, así como la producción de subjetividades que desde estos marcos se generan, en aras de visibilizar los procesos de marginalización e interrumpir en alguna medida los mecanismos de desigualdad y dominación, incorporando la denuncia de dichas condiciones en los procesos investigativos realizados.

La instalación de las jóvenes y niñas en jerarquías localizadas de racialización y generización, hace necesario problematizar las lecturas simplistas que afirman que las condiciones de opresión y subalternización asociadas al género han sido superadas, por la incorporación de las mujeres al mercado laboral o a los procesos educativos. Sin desconocer los avances de las sociedades contemporáneas en torno a estos temas, sigue siendo importante afianzar el análisis sobre los poderes hegemónicos que han relegado a determinados sujetos como niñas y jóvenes racializadas, a los márgenes de las ciudades y de los sistemas sociales.

Como lo traza Yacila en su relato:

En el que los hombres siempre son como la figura, entonces alguien va a buscar a Afro** y va y busca a este chico o a este chico, pero nunca buscan a una chica, ¿Cierto? Y pareciera que Afro** fueran los hombres, que las mujeres estuviéramos más invisibles. Entonces empezaron a generarse ese tipo de interrogatorios, pero se generaron precisamente porque Afro** ya había empezado a abrir como un poco esa capacidad de mirar las dimensiones políticas de las cosas ¿Cierto? Entonces con las amigas empezamos a hablar del asunto y era, pero ve, pero es que nosotras, pues los pelados a veces nos dicen que participemos más, que tomemos el grupo como si fuera nuestro, pero uno siente que realmente las condiciones no están dadas, que eso es más como de dientes para afuera, entonces con las peladas, con varias compañeras del grupo nos empezamos a reunir aparte (Yacila, comunicación personal 2015).

Lo anterior implica el desarrollo de autonomías en la producción del conocimiento, en los y las investigadores/as para propiciar la crítica al interior de la misma institución académica y la vez favorecer nuevos medios de democratización del conocimiento, de construcción con los y las comuni- 
dades, de generar cada vez más investigaciones en colabor o en las que por lo menos se interroguen y reestructuren los formatos en los que tradicionalmente se ha hecho ciencia y para que grupos y sujetos como las niñas y jóvenes afrodescendientes o indígenas sean visibles desde sus experiencias y conocimientos en estos espacios. Espacios en los que ellas mismas comienzan a generar rupturas, búsquedas y visibilidades:

Entonces en esa medida no nos sentimos todavía como reconocidas del todo dentro del grupo y dentro de la sociedad. Y eso no va a pasar hasta que nosotras no generemos como espacios para eso, entonces ahí, decidimos crear un espacio aparte donde nosotras nos reuniéramos de vez en cuando y habláramos de las cosas que pasaban al interior del colectivo, pero también de cosas que nosotras sentíamos como mujeres y también para leer, "mira, leamos de esto, hagamos..." y empezamos todas como con esa inquietud y empezamos a leer sobre el feminismo, primero era el feminismo en general, luego alguien nos recomendó que leyéramos a Ángela Davis, después, y así fuimos encontrando un montón de cosas (Yacila, comunicación personal 2015).

Ese encontrarse con un montón de cosas desde la teoría para conectarla con la experiencia concreta de vida, para generar espacios de participación y pensamiento distintos en los que sus voces comiencen a ser visibles, son parte de las luchas que muchas mujeres jóvenes afrodescendientes se encuentran dando en Medellín y en otros lugares de Colombia y América Latina, conscientes de la importancia de sus malestares, exploraciones y movimientos vitales y conceptuales, como forma de apuntalar y transformar el mundo en el que viven.

Como lo plantea Rivera Cusicanqui (2010):

El desafío de esta nueva autonomía reside en construir lazos sur-sur que nos permitan romper los triángulos sin base de la política y la academia del norte. Construir nuestra propia ciencia -en un diálogo entre nosotros mismosdialogar con las ciencias de los países vecinos, afirmar nuestros lazos con corrientes teóricas de Asia y África, y enfrentar los proyectos hegemónicos del norte con la renovada fuerza de nuestras convicciones ancestrales (p. 73).

Que sin centrarse en radicalismos y sin desconocer las posibilidades del conocimiento construido en otras latitudes, pueda interrogársele y a su vez explorar el conocimiento situado latinoamericano y generar aportes que contribuyan a la transformación de las realidades sociales, a establecer nue- 
vos escenarios pedagógicos y académicos y a reconocer que los mismos movimientos sociales latinoamericanos construyen y han construido históricamente conocimiento. El pensamiento descolonizador lleva a afirmar los lazos desde el dialogo con otros y otras construyendo una práctica académica crítica que incorpore a los intelectuales indígenas, a los intelectuales afros, a las niñas, niños y jóvenes que hacen ciencia y a muchos otros/as, la visión y las voces de las comunidades latinoamericanas, de las resistencias y narrativas colectivas de los y las jóvenes, de los niños/as, en general de los sujetos y colectivos sociales.

La población afrodescendiente o indígena, así como otros grupos sociales, no pueden seguir siendo vistos desde la ciencia racial instrumental y las jerarquizaciones de los Estados modernos, especialmente cuando ellos y ellas pueden contribuir de manera decidida en la construcción social y en la transformación de la misma, como lo reflexiona Yacila:

Entonces aquí hay mucha población afro que está muy bien preparada, muy bien cualificada, gente con, afros con situaciones económicas importantes. Si bien no voy a decir que la percepción de diferencia racial ha desaparecido acá, no diría eso nunca, diría que se ve de una manera diferente, pero por lo menos una parte de la gente tiene conciencia de que una persona negra está en las capacidades de hacer lo mismo que hace una persona blanco-mestizo, aunque ellos no sé lo permitan, que es otra cosa, me parece, o sea para la gente está incorporado en su psique que el negro es inferior, simplemente por ser inferior, pero saben que el negro tiene las capacidades de hacer lo mismo que ellos hacen, yo lo siento más o menos así (Yacila, comunicación personal 2015).

Descolonizar el pensamiento, retomando lo que expone Yacila, se convierte en una tarea prioritaria para la sociedad en general, especialmente porque pensar en generar arquitecturas situadas alrededor de la construcción de paz, implica el reconocimiento intercultural y el respeto por las diferencias, pero además de manera particular para los investigadores/as y académicos/ as, dado que las clasificaciones raciales se encuentran inscritas en los sentimientos y creencias de unos sujetos como inferiores y en la superioridad moral de otros sujetos y sus formas de conocimiento y subjetividades. Esto debe ser constantemente interpelado y rebatido desde las discursividades y prácticas, para lograr fisuras y transformaciones en el orden de la colonialidad racializada y patriarcal dominante, para que efectivamente pueda generarse una construcción de paz que proponga un giro epistémico, así como comprensiones simbólicas mucho más amplias y dialógicas del mundo. 
Ahí precisamente el escenario investigativo se nutre desde apuestas metodológicas y epistemológicas diversas que reconfiguran las tradicionales posiciones y ubicaciones de los sujetos en estos procesos, como un aporte que desde este mismo escenario puede realizarse a la construcción de paz, ubicándose desde el reconocimiento de las memorias corporalizadas y territorializadas como instancia ético política.

\section{¿Y qué es eso de la colabor?}

Preguntarse entonces por otras formas de construir conocimiento lleva entonces a la colabor como posibilidad de cuestionar las narrativas y discursos eurocentricos instituidos aun en nuestros territorios, desde una preocupación sobre lo que pasa y nos pasa, en lo que es importante resaltar lo planteado por Quijano (2007, p. 94) en cuanto a que "El eurocentrismo, por lo tanto, no es la perspectiva cognitiva de los europeos exclusivamente, o sólo de los dominantes del capitalismo mundial, sino del conjunto de los educados bajo su hegemonía" lo que indudablemente coincide con el colonialismo interno presentado por Rivera Cusicanqui (2010). Por lo tanto, la labor de descolonización no es un asunto para ser puesto en otros/as, sino que inicia con nosotros/as mismos como sujetos y como investigadores/as, precisamente porque la perspectiva cognitiva en la que hemos sido educados ha naturalizado la visión eurocentrica del mundo como la única narrativa posible.

Desde los colectivos y grupos de niños, niñas y jóvenes se han venido forjando acciones de resistencia como la capoeira, el grafiti, el hip hop y muchas otras expresiones artísticas y políticas, que han permitido que emerjan nuevos sentidos y prácticas, acciones políticas diversas de descolonización y agenciamiento que deben ser cada vez más visibles. La búsqueda metodológica como pista para profundizar en cómo lograr acercarse a jóvenes, niñas y niños, desde una perspectiva en la que no sólo sean partícipes de la investigación, sino que la misma sea configurada y reconfigurada a partir del encuentro con ellas, ellos y sus realidades concretas; reconociendo las opacidades y los silencios que en muchos casos frente a sus narrativas se ha generado desde el conocimiento científico, se establece en las articulaciones epistemológicas y políticas de la colabor y la decolonialidad como alternativas razonables, que pueden además contribuir a la emancipación y construcción de paz, desde la denuncia y contribución a la transformación de las opresiones. 
Adicionalmente y como lo establece Cerda (2014, p. 1) "El enfoque decolonial puede ser visto también como un programa de investigación y acción, de producción de conocimientos y de toma de posición política, que permanece abierto y en construcción" y que abre a las investigaciones a la pluralidad, a la diferencia, a la crítica del conocimiento instaurado, pero además a la visibilización de las voces de los sujetos subalternizados como los niños, niñas y jóvenes, que históricamente han venido siendo invisibilizados. De todas formas, es importante aclarar que esto no puede verse como un rescate fundamentalista que pone un esencialismo en ellos y ellas, sino que por lo contrario, propone como centro de la cuestión las posibilidades alternativas de conocimientos, subjetividades y resistencias que desde la episteme de frontera como las denomina Castro Gómez (2007), se han venido históricamente generando y que se encuentran en niñas, jóvenes y niños como sujetos que también poseen saberes que pueden poner en diálogo intergeneracional e intercultural con otros y otras.

Al respecto entonces vale la pena preguntarse ¿A qué nos referimos cuando hablamos de colabor? Especialmente cuando los esfuerzos por descolonizar el pensamiento realmente inician desde las propias prácticas, desde la problematización de las categorías investigativas y de las certezas instaladas. Efectivamente investigar desde una perspectiva decolonial y en colabor no sólo implica rupturas metodológicas y epistemológicas, sino que también se convierte en una apuesta ética y política de quienes quieren replantear las formas tradicionales de realizar investigación y de aproximarse a los sujetos con los que se investiga.

La colabor como la conciben Duarte y Berrio (s.f., p. 3) es "aquella en la que el planteamiento del problema, el desarrollo de la pesquisa, la elaboración de los productos y la devolución de los resultados se realiza en conjunto, ninguna de las autoras hemos hecho estrictamente investigación de esta índole", porque implica comprometerse con los actores sociales, con niños, niñas y jóvenes, generando con ellos/as las reflexiones y construcciones en torno a la investigación, lo que no siempre es fácil en medio de una lógica académica e institucional.

Pero más allá de que se pueda o no establecer una investigación en los términos estrictos de la colabor con niños, niñas y jóvenes, el diálogo como lo tratan Duarte y Berrio (s.f.) aparece como un escenario central a las investigaciones desde el interés por cuestionar las relaciones de poder y ejercer vigilancia epistemológica, a la vez que considerar las búsquedas políticas 
de ellos y ellas como centrales, lo que implica relaciones de horizontalidad y reconocimiento mutuo. Relaciones que no necesariamente son fáciles de construir en la cotidianidad por las propias estructuras que alrededor de la ciencia, de la construcción del conocimiento, se tienen desde ambas partes y las posturas adultocentricas asociadas, pero en el que se han presentado avances desde las posibilidades de reflexión ética y deslocalización de los lugares tradicionales de producción científica.

La investigación en colabor además lleva a deconstruir la idea de la academia como el espacio exclusivo de la construcción de conocimiento y pone en un lugar de profunda validez, los agenciamientos que al respecto se dan en los y las jóvenes, en niños y niñas y los inteligentes aportes que ellos y ellas pueden hacer desde su conocimiento a la construcción de paz, a formas distintas de relacionarnos con otros, otras, incluyendo a lo vivo, a los animales, al planeta mismo, que desde perspectivas adultocentricas no logran visualizarse. Esta visión de la paz como un asunto que no se centra exclusivamente en lo antropocéntrico, sino que tiene una base más amplia, aparece especialmente en los niños y niñas en conexión directa con pedagogías y cosmovisiones ancestrales, aunque expresadas en lenguajes distintos, pero, aunque se encuentran ambas visiones presentes en el espacio social, poco reconocimiento ha tenido en las prácticas sociales, así como en los debates, escritos e inserciones del trabajo científico y académico.

En esa medida la pregunta es cómo investigar con ellas/ellos y no sobre ellas/ellos, como además generar como lo plantea Freire $(1973$, p. 3) una comunicación verdadera que no sea "trasferencia, o trasmisión del conocimiento, de un sujeto a otro, sino su coparticipación en el acto de comprender" y poder llegar a esa construcción de ciencia social de la que habla el gran maestro e investigador colombiano Fals Borda (1999, p. 5) "la ciencia se construye socialmente, y que por lo tanto queda sujeta a interpretación, reinterpretación, revisión y enriquecimiento" por parte de todos/as los que participan en el proceso de construcción del conocimiento.

La mayor dificultad reside en cómo generar una apropiación social del conocimiento que no sólo se de en la vía de los grupos científicos hacia los niños, niñas y jóvenes, sino que de manera recíproca también se reconozca y respete el conocimiento de ellas y ellos, especialmente frente a un tema crítico en Colombia (lugar geopolítico desde el que se escribe este texto) y en otros países de América Latina, como el de la construcción de paz y que el mismo sea material de lectura, reflexión, etc de los grupos científicos, no 
sólo desde la información generada a partir de las técnicas utilizadas, sino desde el encuentro intersubjetivo que se da en todos los momentos de la indagación. Esto que aún se efectúa muy poco, constituye un desafío que esta manera de producir conocimiento, forja y contribuye al debate sobre los procesos de validación y generación del mismo.

Una postura epistemológica desde la colabor y específicamente en torno a la construcción de paz, tendría que propiciar una reflexión sobre las posiciones de sujeto de quienes investigan, pero además sobre las relaciones que se establecen en el marco de las investigaciones, como operan esas preguntas de investigación en el contexto de cada país y de América Latina y la consideración del conocimiento cómo una construcción social que no desconoce el saber de los otros/as, pero también la colonialidad que se ha establecido en esa construcción y en la universidad misma. Aquí resulta clave lo que al respecto sugiere Castro Gómez (2007):

La función de la universidad hoy día ya no es educar sino investigar, lo cual significa: producir conocimientos pertinentes. Los profesores universitarios se ven abocados a investigar para generar conocimientos que puedan ser útiles a la biopolítica global en la sociedad del conocimiento. De este modo, las universidades empiezan a convertirse en microempresas prestadoras de servicios (p. 85).

Por lo que es fundamental preguntarse ¿Cómo generar investigación desde la universidad que se resista a esta utilización del conocimiento? ¿Cómo ir más allá de la participación concreta de jóvenes, niños y niñas como generadores de información? La apuesta de Fals Borda (1999) es que tanto el investigador como los participantes de la investigación sean vistos como seres sentipensantes o como lo plantea Alejandro Cerda (2013) la búsqueda de una investigación vinculada que abre la posibilidad de que elementos de esos sujetos con los que investigo -niñas, niños y jóvenes- hagan parte del proyecto de vida del investigador/a.

Este último elemento parece realmente provocador porque bosqueja además preguntas sobre el papel de los intelectuales en la sociedad, pregunta central que puede retomarse en lo planteado por Fals Borda (1999):

¿Podríamos entonces ser al mismo tiempo intelectuales estudiosos y agentes del cambio con el fin de cooperar en este movimiento intelectual y político, dirigido a levantar la bandera del poder y la autonomía populares, para de- 
fender la vida en todas sus formas, y para adelantar la construcción de una ciencia útil y pertinente? (p. 17).

Esto tendría que generarse desde el mismo inicio de la investigación, desde el entrecruzamiento de las distintas visiones de mundo, pero también como lo enuncia Lenkersdorf (2008) distintas cosmoaudiciones.

Vale la pena retomar además lo planteado por las feministas, especialmente Haraway (1995, pp. 187-188) en torno al conocimiento como forma de contribuir a la descolonización desde la posibilidad de situarlos histórica, geográfica y contextualmente:

Los conocimientos situados son herramientas muy poderosas para producir mapas de conciencia para las personas que han sido inscritas dentro de las marcadas categorías de raza y de sexo, tan exuberantemente producidas dentro de las historias de las dominaciones masculinistas, racistas y colonialistas. Los conocimientos situados son siempre conocimientos marcados.

que pueden llevar a la consideración sobre la propia situación en el mundo como investigadores/as, como sujetos con cuerpo, género, historia y motivaciones particulares frente a lo que realizamos, y sobre los escenarios de violencias que se reproducen desde las representaciones que se efectúan de los otros en los procesos investigativos o las formas en que desde ahí se puede contribuir a la emancipación y construcción de paz.

La vigilancia epistemológica implica también preguntarse de manera crítica sobre la propia construcción de teoría como científicos sociales, poner en cuestión también las categorías que se están construyendo y así como lo explicita Rivera Cusincanqui (1990) el reconocer la intraducibilidad lingüística y cultural, propia de una relación asimétrica entre individuos y culturas cuyo horizonte cognoscitivo es diametralmente opuesto, forma parte del dilema ético de las ciencias contemporáneas en las que el conocimiento convertido en instrumento de poder y prestigio, puede volcarse en contra de las necesidades e intereses de la sociedad misma, de los sujetos con los que se investigó y el investigador transformarse en agente de la opresión y la subalternización.

Rivera Cusicanqui (1990) además traza la necesidad de mirar la investigación como el escenario en que dos sujetos reflexionan sobre su experiencia y sobre la visión que cada uno tiene del otro, generando así un pacto de confianza que permite la incubación de narrativas en cuyo proceso se 
va transformando lo acontecido para descubrir lo significativo y que genera otras lógicas de lo investigativo.

Esto implica entender la investigación como una dialéctica de polos pensantes (Rivera Cusicanqui, 1990) porque no necesariamente las relaciones investigativas y la propia construcción de paz están exentas de tensiones, por lo contrario, se encuentran habitadas por las posibilidades, pero también por obstáculos, desencuentros, dadas las asimetrías de la relación misma, pero también porque las expectativas y motivaciones de cada una de las partes pueden ser diferentes. Precisamente ese es también uno de los mayores desafíos que tiene la investigación en colabor dado que pone al investigador en un rol distinto al que tradicionalmente se ha instalado y le genera desde ahí y desde el diálogo horizontal, otras perspectivas.

Adicionalmente implica pensar a los niños, niñas y jóvenes no como especificidades homogéneas, sino desde su pluralidad, resaltando que a su interior también existen sujetos con intereses investigativos, propiciándoles el situarse más allá de las generalizaciones normalizantes que los ubica en la minoría de edad. Esto pone en contexto la asimetría que ya nos mencionaba Rivera Cusicanqui (2010) en los mismos procesos de muchas de las apuestas investigativas que se realizan, a pesar de que existe un interés discursivo y político en generar alternativas de construcción de conocimiento, no es fácil necesariamente descolonizar el pensamiento y las certezas metodológicas e investigativas instaladas; ese es a la larga el gran reto, especialmente si se quiere construir paz de manera dialógica, contando con niños, niñas y jóvenes, quienes:

Ponen su acento en lo alternativo al ampliar el significado y uso del cuerpo como primer territorio de poder y resistencia, para vencer el miedo y como expresión de paz; la deconstrucción de los derechos desde una perspectiva comunitaria y de des-colonización. Los jóvenes y las jóvenes usan el arte, la estética y lo lúdico como medios de creación y expresión para desnaturalizar lugares y sentidos de enunciación, y mostrar formas de actuaciones plurales y sensibles que no se agotan en la razón (Alvarado, Borelli y Vommaro, 2012, p. 59).

De esta manera el cuerpo y el uso de otros lenguajes para escenificar su acción política, los sueños, opacidades y posibilidades de niños, niñas y jóvenes, propician otras interlocuciones, mucho más heteroglósicas y plurales, que interpelan las formalizadas y adultocentricas agendas académicas, 
generando desplazamientos y roles más diversos y menos estandarizados en los escenarios sociales y los mismos procesos investigativos.

\section{Reflexiones finales}

Tal cual como lo enuncia Walsh (2013, p. 24):

Son estos momentos complejos de hoy que provocan movimientos de teorización y reflexión, movimientos no lineales sino serpentinos, no anclados en la búsqueda o proyecto de una nueva teoría crítica o de cambio social, sino en la construcción de caminos - de estar, ser, pensar, mirar, escuchar, sentir y vivir con sentido o horizonte de(s)colonial.

que ilumine el camino de la indagación científica pero también las vidas y la cotidianidad de todos los sujetos que hacen parte de las investigaciones, a la sociedad latinoamericana con sus problematizaciones, realidades y anhelos de construcción de paz como un camino posible desde la edificación de espacios de participación, encuentro, reflexión y acción política, lo que va de la mano con la interrupción de las condiciones de opresión y marginalización de quienes han sido históricamente excluidos de los debates públicos y la construcción social, incluyendo a niñas, niños y jóvenes.

Las ciencias sociales han puesto mucho de sus sentidos y apuestas en las construcciones orales y narrativas, generando preguntas por las subjetividades que desde lo biográfico se van configurando, las rupturas que también desde ahí en la memoria y la experiencia vivida se producen, el encuentro con los vacíos y los silencios. Responder a los silencios, pero también a los retos y demandas que la escucha atenta genera, a las enunciaciones políticas de los sujetos y a la cercanía y posición ética y política del investigador/a con el compromiso de comprensión de las distintas realidades, pero también con su transformación si es necesario, es la gran búsqueda de la investigación en colabor con una perspectiva decolonial, como aporte significativo a las múltiples paces, las que deberán construirse en la particular vivencia del caso colombiano alrededor del conflicto armado y político, pero también las que en otros países tendrán que generarse alrededor de las dolorosas experiencias de las dictaduras o al interior de las familias y escuelas, en suma, en las sociedades latinoamericanas en su singularidad y diversidad. 
Esto implica el reconocimiento por parte de los investigadores/as sobre el que:

Las luchas sociales también son escenarios pedagógicos donde los participantes ejercen sus pedagogías de aprendizaje, desaprendizaje, reaprendizaje, reflexión y acción. Es sólo reconocer que las acciones dirigidas a cambiar el orden del poder colonial parten con frecuencia de la identificación y reconocimiento de un problema, anuncian la disconformidad con y la oposición a la condición de dominación y opresión, organizándose para intervenir; el propósito: derrumbar la situación actual y hacer posible otra cosa (Walsh 2013, p. 29).

Pero además salir de la falta, de las visiones eurocéntricas, reconocer la potencia del pensamiento racializado, de jóvenes, niñas y niños, de las experiencias particulares como latinoamericanos y del aporte necesario de los académicos en diálogo con ellas y ellos para la transformación de las sociedades, forma parte de los desafíos que el pensamiento decolonial y la investigación en colabor plantean, pero que es substancial entender y extender en aras de una construcción de paz que no implique negar el conflicto social pero si tramitarlo de maneras distintas a la violencia. Esto implicaría desde luego subjetivarse de otro modo, recorrer el mundo de una manera diferente, subvirtiendo las normas, cuestionando el canon académico, alejándose de la mirada propia y lejana del déficit, de la carencia y valorar las subjetividades barrocas como lo plantea Santos (2009) que permitan continuar luchas emancipatorias y que propicien así que la descolonización sea realmente una práctica vital e investigativa, un aporte fundamental a la construcción de paces sostenibles y duraderas en las que niños, niñas y jóvenes en dialogo intergeneracional sean también protagonistas.

Las investigaciones y sus planteamientos epistemológicos pueden ser campos de visibilización y creación, de prácticas políticas transformadoras en la medida en que puedan acercarse a los jóvenes y niños desde sus propios lenguajes, con metodologías diversas e innovadoras que disminuyan las asimetrías, involucrándose los investigadores mismos en el proceso de construcción de conocimiento desde subjetividades que se encuentran, reconociendo los tránsitos disimiles de estas subjetividades por el deseo, los miedos y las pasiones, pero sobre todo el ineludible compromiso de la experiencia investigativa como una práctica política y ética, que puede contribuir a transformar la sociedad de la que se es parte o a mantener las subordinaciones y opresiones existentes. 
Esta resonancia investigativa y de vida implica como lo plantea Lenderskoff (2008, p. 25) aprender a escuchar, porque "el escuchar, pues, nos abre las puertas, para entrar en otra cultura. Al hablar con la gente, nos pueden abrir su corazón, explicar sus problemas y alegrías y hacernos participar en el mundo que viven. A la vez nos pueden cuestionar e interpelar si aceptamos sus preguntas", y así definir rutas críticas, de dialogo, de interpelación y aprendizajes mutuos, de transformación política y social, de explicitación de posturas éticas que propicien nuevos sentidos frente a la producción de conocimiento y la emancipación social, necesaria para superar las condiciones históricas de opresión y ampliar los procesos pacifistas y pacíficos más allá de lo antropocéntrico, escuchando a los niños, niñas y jóvenes, propiciando a su vez que ellos y ellas, con otros y otras también puedan escucharse, afianzando y visibilizando las acciones cotidianas, concretas de construcción de paz que ellos y ellas ya vienen realizando, pero también las que pueden ampliar y seguir construyendo.

Finalmente, los escenarios investigativos desde estas perspectivas, implican también la posibilidad para los investigadores (as) de generar un proceso reflexivo sobre sí mismos, de escucharse y observar las transformaciones subjetivas que se generan en ella o él y en los otros con los que comparte esa aventura, permitiéndose el asombro y el dejarse llevar de la mano de los niños, niñas y jóvenes, por sus historias, comprensiones sociales y las acciones que cotidianamente realizan, en aras de -con ellos y ellas- realizar fisuras en lo establecido y avanzar en el empoderamiento de todos los participantes de la investigación, en nuevas rutas de resistencia e insurgencia, de caminos alternativos hacia las paces cotidianas que se generan desde el compromiso ético y apasionado por los otros, por el mundo.

\section{Bibliografía}

Alvarado, S.V., Borelli, S. y Vommaro, P. (2012). GT Juventud y prácticas políticas en América Latina: comprensiones y aprendizajes de la relación juventud-política-cultura en América Latina desde una perspectiva investigativa plural en jóvenes, politicas y culturas: experiencias, acercamientos $y$ diversidades. 1a ed. Argentina: Homo Sapiens Ediciones, CLACSO.

Alexander, M. J. y Mohanty, C. (2004). Genealogías, legados, movimientos En: Otras inapropiables. España: Editorial Traficante de sueños. 
Antón, J., Bello, Á., Del Popolo, F., Paixão, M. \& Rangel, M. (2009). Afrodescendientes en América Latina y el Caribe: del reconocimiento estadístico a la realización de derechos. CEPAL, UNFPA.

Carosio, A. (2010). El saber desde las mujeres. Los estudios de género y de las mujeres en Venezuela. En: A. Delgado y M. Camacaro (Coords.), La Academia en sintonía de género: una discusión impostergable. Venezuela: Universidad de Carabobo.

Carneiro,S.(2009).Ennegrecerelfeminismo.Dipsonibleen:Negracubanateníaqueser: http://negracubana.nireblog.com/post/2009/07/28/sueli-carneiro-enne greceral-feminismo

Castro-Gómez, S. y Grosfoguel, R. (2007). Prólogo. Giro decolonial, teoría crítica y pensamiento heterárquico en El giro decolonial: reflexiones para una diversidad epistémica más allá del capitalismo global. Bogotá: Siglo del Hombre Editores, Universidad Central, Instituto de Estudios Sociales Contemporáneos y Pontificia Universidad Javeriana, Instituto Pensar.

Castro-Gómez, S. (2007). Decolonizar la universidad. La hybris del punto cero y el diálogo de saberes. En: S. Castro-Gómez y R. Grosfoguel (Eds.), El giro decolonial: reflexiones para una diversidad epistémica más allá del capitalismo global (pp. 79-93). Bogotá: Siglo del Hombre Editores, Universidad Central, Instituto de Estudios Sociales Contemporáneos y Pontificia Universidad Javeriana, Instituto Pensar.

Cerda, A. (2013). Diversidad epistemológica: descolonización y saberes emergentes. En: En el juego de los espejos. Multi, inter, transdisciplina e investigación cualitativa en salud (pp. 103-120). México: UAM-X.

(2014). "Clase 2: Investigación hacia la descolonización o en co-labor. Referencias epistemológicas”. Documento CLACSO.

Curiel, O. (2009). Ponencia presentada en el Primer Coloquio Latinoamericano sobre Praxis y Pensamiento Feminista realizado en Buenos Aires en junio de 2009, organizado por el grupo Latinoamericano de Estudios, Formación y Acción Feminista (GLEFAS) y el Instituto de Género de la Universidad de Buenos Aires.

Del Popolo, F., López, M. \& Acuña, M. (2009). Juventud indigena y afrodescendiente en América Latina: inequidades sociodemográficas y desafios de políticas. Centro Latinoamericano y Caribeño de Demografía (CELADE)División de Población de la CEPAL.

Duarte Bastián Á.I. y Berrío Palomo, L.R (s.f). Saberes en diálogos. Alianza entre mujeres indígenas y académicas en la construcción de conocimientos. 
En: Conocimientos y prácticas políticas: reflexiones desde nuestras prácticas de conocimiento situadas. México, CIESAS.

Fanon, F. (1963). Los condenados de la tierra. Colectivo Editorial Último Recurso. Fals, Borda, O. (1999). Orígenes universales y retos actuales de la IAP (investigación acción participativa). Análisis Político, 38, septiembre/diciembre.

Freire, P. (1973) ¿Extensión o Comunicación? La concientización en el medio rural. Montevideo, Uruguay: Ed. Siglo XXI.

Habermas, J. (2002). Ciencia y técnica como “ideología”. Madrid. Editorial Tecnos. Harding, S. (1998). ¿Existe un método feminista? Documento PDF. Disponible en: http://148.206.107.15/biblioteca_digital/capitulos/81-2350ske.pdf

Haraway, D. (1995). Ciencia, cyborgs y mujeres: la reinvención de la naturaleza. Madrid: Ediciones Cátedra S.A.

Kant, I. [1975] (2005). Sobre la paz perpetua. Madrid: Editorial Tecnos.

Kuhn, T. [1971] (2011). La estructura de las revoluciones cientificas. México: Fondo de Cultura Económica.

Lakatos, I. [1983] (1993). La metodología de los programas de investigación cientifica. Madrid: Editorial Alianza.

Lenkersdorf, C. (2008). Aprender a escuchar: enseñanzas maya tojolabales. México: D.F. Plaza y Valdés S.A.

Mayer, L. (2015). Feminización y masculinización del espacio escolar. La necesidad del "hombre": Un análisis de las estrategias para la prevención de la conflictividad escolar en escuelas secundarias de la ciudad de Buenos Aires. En: R. Unda Lara, et al., Socialización escolar: Procesos, experiencias y trayectos. 1ra edición: Universidad Politécnica Salesiana, CINDE: Fundación Centro Internacional de Educación y Desarrollo Humano, Universidad de Manizales, CLACSO: Consejo Latinoamericano de Ciencias Sociales, Editorial Universitaria Abya-Yala.

Mignolo, W. (2003). Historias locales, diseños globales: colonialidad, conocimientos subalternos y pensamiento fronterizo. Madrid: Editorial Akal.

Mohanty, C. (2008). Bajo los ojos de occidente. Academia Feminista y discurso colonial. En: Liliana Suárez Navas y Aída Hernández (Eds.), Descolonizando el feminismo: Teorías y prácticas desde los márgenes. México: Ed. Cátedra.

Popper, K. [1962] (2008). La lógica de la investigación cientifica. Madrid: Editorial Tecnos.

PNUD (2013). Informe sobre Desarrollo Humano 2013. El ascenso del Sur: Progreso humano en un mundo diverso. Recuperado en http://www.undp.org/ content/dam/venezuela/docs/undp_ve_IDH_2013.pdf 
Quijano, A. (2007). Colonialidad del poder y clasificación social. En: Santiago CastroGómez y Ramón Grosfoguel (Eds.), El giro decolonial: reflexiones para una diversidad epistémica más allá del capitalismo global. Bogotá: Siglo del Hombre Editores; Universidad Central, Instituto de Estudios Sociales Contemporáneos y Pontificia Universidad Javeriana, Instituto Pensar.

(2014). Cuestiones y horizontes: de la dependencia histórico-estructural a la colonialidad/descolonialidad del poder. 1a ed. Buenos Aires: CLACSO.

Rivera Cusicanqui, S. (1990). El potencial epistemológico y teórico de la historia oral: de la lógica instrumental a la descolonización de la historia. Temas Sociales, 11, 49-75.

(2010). Ch'ixinakax utxiwa: una reflexión sobre prácticas y discursos descolonizadores. 1a ed. Buenos Aires: Tinta Limón.

Sousa Santos, B. de (2003). Crítica de la razón indolente: contra el desperdicio de la experiencia. Para un nuevo sentido común: la ciencia, el derecho y la política en la transición paradigmática. España: Editorial Desclée de Brouwer S.A.

(2006). Conocer desde el Sur: Para una cultura política emancipatoria. Lima. Fondo Editorial de la Facultad de Ciencias Sociales / Unidad de Post Grado

(2009). Una epistemología del sur: la reinvención del conocimiento y la emancipación social. México. Siglo XXI: CLACSO.

Segato, R. L. (2003). Las estructuras elementales de la violencia. Buenos Aires, Bernal: Universidad Nacional de Quilmes.

Schiwy, F. (2002). ¿Intelectuales subalternos? Notas sobre las dificultades de pensar en diálogo intercultural en Indisciplinar las ciencias sociales. Geopoliticas del conocimiento y colonialidad del poder: perspectivas desde lo andino. Quito: Universidad Andina Simón Bolívar, Abya-Yala.

Walsh, C., Schiwy, F. \& Castro Gómez, S (2002). Indisciplinar las ciencias sociales. Geopolíticas del conocimiento y colonialidad del poder: perspectivas desde lo andino. Quito: Universidad Andina Simón Bolívar, Abya-Yala.

Walsh, C. (2013). Pedagogías decoloniales: Prácticas insurgentes de resistir, (re) existir y (re)vivir. Serie "Pensamiento decolonial" Quito: Abya-Yala.

Fecha de recepción: 2016/09/17; fecha de aprobación: 2016/12/03 